ИЗВЕСТИЯ АКАДЕМИИ НАУК ЭСТОНСКОП ССР. ТОМ 30 ФИЗИКА * МАТЕМАТИКА. 1981, № 2

\title{
ОБ ОДНОМ МЕТОДЕ РЕШЕНИЯ ОДНОМЕРНЫХ ВОЛНОВЫХ УРАВНЕНИЙ
}

\section{Введение}

Одномерное волновое уравнение

$$
d^{2} U / d z^{2}+k^{2} n^{2}(z) U=0,
$$

где $U$ - амплитуда монохроматической волны и $n(z)-$ зависящий от координаты $z$, но не зависящий от волнового числа $k$ показатель преломления, имеет решение в замкнутом виде только для некоторых форм зависимости $n(z)$. Многие из этих решений давно известны и хорошо изучены (см., напр., $\left.\left[{ }^{1}\right]\right)$. В статьях $\left[{ }^{2-5}\right]$ были приведены новые случаи волновых уравнений, решаемых в замкнутом виде. В настоящей статье мы изложим другую, более общую методику нахождения подобных уравнений и их решений, причем будут обнаружены новые примеры.

\section{Метод}

Излагаемый ниже метод является частной реализацией (в применении к одномерным волновым уравнениям) общего преобразования, связывающего решения двух линейных дифференциальных уравнений второго порядка (см. $\left[{ }^{6}\right]$, с. 151$)$. Насколько нам известно, до работ $\left[{ }^{2-5}\right]$ этот метод в теории волновых уравнений не применялся.

Будем искать решение уравнения (1) в виде

$$
U(z)=F(z) \Theta(G(z)),
$$

где $\Theta(G)$ удовлетворяет уравнению

$$
d^{2} \Theta / d G^{2}+p(G) \Theta=0
$$

с некоторой, пока неконкретизируемой функцией $p(G)$. Подставляя решение (2) в уравнение (1), с учетом (3) находим

$$
\left(2 F^{\prime} G^{\prime}+F G^{\prime \prime}\right) \Theta^{\prime}+\left(F^{\prime \prime}-F G^{2} p+k^{2} n^{2} F\right) \Theta=0,
$$

где штрих у $G$ и $F$ означает производную по $z$, а у $\Theta$ по $G$. Так как $\Theta$ определяется уравнением (3), то удовлетворить независимому уравнению (4) возможно только приравняв коэффициенты нулю. Итак,

$$
2 F^{\prime} G^{\prime}+F G^{\prime \prime}=0
$$

H

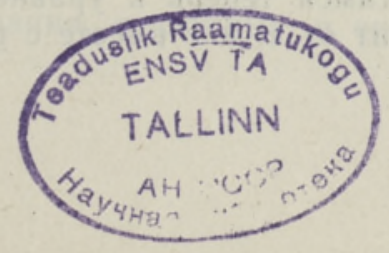




$$
-F G^{2} p+F^{\prime \prime}+k^{2} n^{2} F=0 .
$$

Интегрируя первое из этих уравнений, находим

$$
F=G^{\prime-1 / 2} \text {. }
$$

Подставляя это выражение во второе уравнение, получаем

$$
k^{2} n^{2}=G^{\prime \prime \prime} / 2 G^{\prime}-3 G^{\prime \prime 2} / 4 G^{\prime 2}+p(G) G^{\prime 2} .
$$

Далее положим

$$
G^{\prime} \equiv d G / d z=h^{-1} y^{2}(z) \Psi_{1}^{2}(G),
$$

где $y(z)$ и $\Psi_{1}(G)$ - две новые функции, а $h-$ постоянная размерности длины, означающая в конкретных задачах обычно толщину слоя. Отсюда находим производные $G^{\prime \prime}$ и $G^{\prime \prime \prime}$ в виде:

$$
G^{\prime \prime}=2 h^{-2} y^{4} \Psi_{1}^{3} \Psi_{1}^{\prime}+2 h^{-1} y y_{1}^{\prime} \Psi_{1}^{2}
$$

И

$$
\begin{gathered}
G^{\prime \prime \prime}=6 h^{-3} y^{6} \Psi_{1}^{4} \Psi_{1}^{\prime 2}+2 h^{-3} y^{6} \Psi_{1}^{5} \Psi_{1}^{\prime \prime}+12 h^{-2} y^{3} y^{\prime} \Psi_{1}^{3} \Psi_{1}^{\prime}+ \\
+2 h^{-1} y y^{\prime \prime} \Psi_{1}^{2}+2 h^{-1} y^{\prime 2} \Psi_{1}^{2}
\end{gathered}
$$

где штрих у $y$ означает производную по $z$, а у $\Psi_{1}$ по $G$. Подставляя эти выражения в уравнение (8), находим

$$
k^{2} n^{2}=y^{\prime \prime} / y-2 y^{\prime 2} / y^{2}+G^{\prime 2}\left(\Psi_{1}^{\prime \prime} / \Psi_{1}+p\right) .
$$

Далее введем еще две новые функции, пока произвольные, $f(y)$ и $q(G)$ и потребуем выполнения следующих уравнений:

$$
d^{2} \Psi_{1} / d G^{2}+q(G) \Psi_{1}=0
$$

и

$$
y^{\prime \prime} / y-2 y^{\prime 2} / y^{2}=f(y) / h^{2} .
$$

Тогда соотношение (12) примет вид

$$
k^{2} n^{2}=f(y) / h^{2}+G^{\prime 2}(p(G)-q(G)) .
$$

Общее решение уравнения (14) таково:

$$
z / h=\int y^{-2}\left[C+2 \int y^{-3} f(y) d y\right]^{-1 / 2} d y,
$$

где $C-$ постоянная интегрирования. Интегрируя с учетом (13) уравнение (9), находим

$$
\Psi_{2}(G) / \Psi_{1}(G)=h^{-1} \int y^{2}(z) d z
$$

где $\Psi_{2}$ - линейно независимое от $\Psi_{1}$ решение уравнения (13), причем оба нормированы так, что вронскиан равен единице:

$$
\Psi_{1} \Psi_{2}^{\prime}-\Psi_{1}^{\prime} \Psi_{2}=1 .
$$

Из формул (16) и (17) следует также

$$
\Psi_{2}(G) / \Psi_{1}(G)=\int\left[C+2 \int y^{-3} f(y) d y\right]^{-1 / 2} d y .
$$

Обратимся теперь к уравнению (15). Так как $n$ зависит от $z$, но не зависит от $k$, а $z$ вместе с $y$ зависит, согласно формуле (19), от $G$, 
то ни $y$, ни $G$ не могут зазвисеть от $k$. В силу уравнений (13) и (14) $f(y)$ и $q(G)$ тоже не могут зависеть от $k$. Следовательно, единственной функцией, могущей дать множитель $k^{2}$ в левую часть уравнения (15), является $p(G)$. Согласно сказанному положим

$$
p(G)=q(G)+k^{2} h^{2} N^{2}(G)-h^{-2} f(y) G^{\prime-2},
$$

где $N(G)$ - еще одна функция, не зависящая от $k$. Иначе, в силу формулы (9) можно написать:

$$
p(G)=q(G)+k^{2} h^{2} N^{2}(G)-f(y) y-4 \Psi-4 .
$$

Кіз формулы (15) теперь следует

или, опять в силу (9),

$$
n=h N(G) d G / d z
$$

$$
n=N(G) \Psi_{1}^{2}(G) y^{2} .
$$

Наконец, уравнение (3) получает вид

$$
d^{2} \Theta / d G^{2}+\left[q(G)+k^{2} h^{2} N^{2}(G)-f(y) y^{-4} \Psi_{1}^{-4}\right] \Theta=0 .
$$

Итак, мы пришли к следующему результату. Заданы три функции $q(G), N(G)$ и $f(y)$. Первую из них следует выбирать так, чтобы уравнение (13) решалось в замкнутом виде, а две остальные - так, чтобы в замкнутом виде решалось уравнение (24). При этом связь между $G$ и $y$ осуществляется формулой (19). Тогда волновое уравнение

$$
d^{2} U / d z^{2}+k^{2} N^{2}(G) \Psi_{1}^{4} y^{4} U=0
$$

имеет замкнутое решение (см. формулы (2), (7) и (9))

$$
U=y^{-1} \Psi-1 \Theta \text {. }
$$

Заметим, что это решение получается в параметрическом виде, т. е. $U$ получается как функция параметра $G$, а не координаты $z$. Показатель преломления (формула (23)) тоже задан как функция $G$. Следовательно, мы должны еще знать зависимость между $G$ и $z$. Для этого имеем формулу (16), определяющую $z$ как функцию $y$. А так как $y$ уже выражено (по формуле (19)) в зависимости от $G$, то мы получаем и $z$
как функцию $G$.

Исключение параметра возможно далеко не всегда, но если исключение невозможно, то часто бывает полезно произвести замену параметра с целью упрощения конечных формул. где

Прежде чем перейти к примерам, отметим важный частный вариант,

$$
f(y)=0 .
$$
Интегралы в формулах $(16)$ и $(19)$ тогда легко вычисляются до конца,
и мы получаем (заменив $\left.C=c^{2}\right)$ :

и

$$
y(z)=(c z / h+d)^{-1}
$$

$$
\Psi_{2} / \Psi_{1}=(a z / h+b)(c z / h+d)^{-1},
$$

где $a, b, c, d-$ постоянные интегрирования, связанные, в силу формулы (18), соотношением 


$$
a d-b c=1 .
$$

Уравнение (13) в этом варианте остается неизменным, а уравнения (24) и $(25)$ и формулы $(23)$ и $(26)$ принимают вид

$$
\begin{gathered}
d^{2} \Theta / d G^{2}+\left[q(G)+k^{2} h^{2} N^{2}(G)\right] \Theta=0, \\
d^{2} U / d z^{2}+k^{2} N^{2}(G) \Psi_{1}^{4}(c z / h+d)^{-4} U=0, \\
n=N(G) \Psi_{1}^{2}(G)(c z / h+d)^{-2}
\end{gathered}
$$

и

$$
U=(c z / h+d) \Psi_{1}^{-1} \Theta .
$$

Последний результат является обобщением полученного в $\left[{ }^{2}\right]$. В самом деле, положив в уравнениях (13) и (31) $q(G)=f(u), N(G)=$ $=h^{-1}, \Psi=s$ и $\Theta=A$, мы получим соответствующие уравнения (6) и (9) статьи $\left[{ }^{2}\right]$. Формулы (29) и (32)-(34) можно несколько упростить. Поскольку из формулы (29), с учетом (30) вытекает

$$
\Psi_{1}(G)(c z / h+d)^{-1}=a \Psi_{1}-c \Psi_{2},
$$

то целесообразно переобозначить $\Psi_{1}$ и $\Psi_{2}$ согласно следующим линейным комбинациям

$$
\begin{array}{r}
a \Psi_{1}-c \Psi_{2} \rightarrow \Psi_{1}, \\
-b \Psi_{1}+d \Psi_{2} \rightarrow \Psi_{2},
\end{array}
$$

причем вронскиан для новых $\Psi_{1}$ и $\Psi_{2}$ по-прежнему равен единице. Тогда получим вместо формул (29) и (32)-(34) следующие более простые формулы

$$
\begin{gathered}
\Psi_{2} / \Psi_{1}=z / h, \\
d^{2} U / d z^{2}+k^{2} N^{2}(G) \Psi_{1}^{4}(G) U=0, \\
n=N(G) \Psi_{1}^{2}(G)
\end{gathered}
$$

и

$$
U=\Psi_{1}^{-1} \Theta
$$

\section{Примеры}

Изложенный выше метод способен дать все или почти все полученные ранее в $\left[{ }^{2-5}\right]$ результаты. Здесь мы приведем новые примеры.

I. В первом примере примем

$$
\begin{gathered}
f(y)=0, \\
q(G)=A^{2}-\left(v^{2}-1 / 4\right) G^{-2}
\end{gathered}
$$

и

$$
N(G)=\left(N_{0}^{2}+\mu^{2} G^{-2}\right)^{1 / 2},
$$

где $A, N_{0}, \mu, v$ - постоянные. Решения уравнения 


$$
d^{2} \Psi / d G^{2}+\left[A^{2}-\left(v^{2}-1 / 4\right) G^{-2}\right] \Psi=0,
$$

удовлетворяющие условию (18), суть

$$
\begin{aligned}
& \Psi_{1}=G_{v}^{1 / 2} Z_{v}^{(1)}(A G), \\
& \Psi_{2}=G_{v}^{1 / 2} Z_{v}^{(2)}(A G),
\end{aligned}
$$

где $Z_{v}{ }^{(1)}$ и $Z_{v^{(2)}}$ - произвольные, линейно независимые функции Бесселя $v$-го порядка с вронскианом, равным $(A G)^{-1}$. Для $\Theta$ имеем уравнение

$$
d^{2} \Theta / d G^{2}+\left[A^{2}+k^{2} h^{2} N_{0}^{2}-\left(v^{2}-k^{2} h^{2} \mu^{2}-1 / 4\right) G^{-2}\right] \Theta=0,
$$

решение которого есть

$$
\Theta=G^{1 / 2} Z_{\left(v^{2}-k^{2} h^{2} \mu^{2}\right)^{1 / 2}}\left(\left(A^{2}+k^{2} h^{2} N_{0}^{2}\right)^{1 / 2} G\right) .
$$

Следовательно, согласно формулам (38) и (40), волновое уравнение

$$
d^{2} U / d z^{2}+k^{2}\left(N_{0}^{2} G^{2}+\mu^{2}\right) Z_{v}^{(1) 4}(A G) U=0
$$

имеет решение

$$
U=Z_{v}^{(1)-1}(A G) Z_{\left(v^{2}-h^{2} h^{2} \mu^{2}\right)^{1 / 2}}\left(\left(A^{2}+k^{2} h^{2} N_{0}^{2}\right)^{1 / 2} G\right),
$$

причем параметр $G$ связан с координатой $z$ соотношением

$$
z / h=Z_{v}^{(2)}(A G) / Z_{v}^{(1)}(A G)
$$

(см. формулу (37)).

В этом примере исключение параметра невозможно, но некоторое упрощение достигается заменой

$$
A G \rightarrow G, \quad N_{0} \rightarrow A^{2} .
$$

Тогда формулы $(48)-(50)$ примут вид:

$$
\begin{gathered}
d^{2} U / d z^{2}+k^{2}\left(A^{2} G^{2}+\mu^{2}\right) \underset{v}{Z(1) 4}(G) U=0, \\
U=\underset{v}{Z(1)-1}(G) Z_{\left(v^{2}-k^{2} h^{2} \mu^{2}\right)^{1 / 2}}\left(G\left(1+k^{2} h^{2} A^{2}\right)^{1 / 2}\right)
\end{gathered}
$$

и

$$
z / h=Z_{v}^{(2)}(G) / Z_{v}^{(1)}(G) .
$$

Этот результат является обобщением примера, приведенного в $\left[{ }^{2}\right]$, и сводится к тому при $\mu=0$. Он обобщает также основной результат статьи $\left[{ }^{4}\right]$, для получения которого следует взять $A=0$.

II. Для второго примера оставим в силе (41) и выберем

$$
q(G)=A+B G^{-2 / 3}+(5 / 36) G^{-2}
$$

и

$$
N(G)=\left(N_{0}^{2}+M_{0}^{2} G^{-2 / 3}\right)^{1 / 2},
$$

где $A, B, N_{0}, M_{0}$ - постоянные. Уравнение

$$
d^{2} \Psi / d G^{2}+\left[A+B G^{-2 / 3}+(5 / 36) G^{-2}\right] \Psi=0
$$

имеет решения 


$$
\begin{aligned}
& \Psi_{1}=\left(G+B A^{-1} G^{1 / 3}\right)^{1 / 2} Z_{1 / 3}^{(1)}\left(\left(A^{-2 / 3} B+A^{1 / 3} G^{2 / 3}\right)^{3 / 2}\right), \\
& \Psi_{2}=\left(G+B A^{-1} G^{1 / 3}\right)^{1 / 2} Z_{1 / 3}^{(2)}\left(\left(A^{-2 / 3} B+\cdot A^{1 / 3} G^{2 / 3}\right)^{3 / 2}\right),
\end{aligned}
$$

где $Z_{1 / 3}^{(1)}$ и $Z_{1 / 3}^{(2)} \quad$ - любые линейно независимые функции Бесселя порядка $1 / 3$ с вронскианом, равным обратному аргументу. Легко убедиться, что условие (18) для $\Psi_{1}$ и $\Psi_{2}$ выполняется. Для $\Theta$ имеем, согласно формулам (31), (55) и (56), уравнение того же вида, что и $(57)$ :

$$
d^{2} \Theta / d G^{2}+\left[A+k^{2} h^{2} N_{0}^{2}+\left(B+k^{2} h^{2} M_{0}^{2}\right) G^{-2 / 3}+(5 / 36) G^{-2}\right] \Theta=0 .
$$

Решение его есть

$$
\begin{gathered}
\Theta(G)=\left[G+\left(B+k^{2} h^{2} M_{0}^{2}\right)\left(A+k^{2} h^{2} N_{0}^{2}\right)^{-1} G^{1 / 3}\right]^{1 / 2} \times \\
\times Z_{1 / 3}\left(\left[\left(A+k^{2} h^{2} N_{0}^{2}\right)^{-2 / 3}\left(B+k^{2} h^{2} M_{0}^{2}\right)+\left(A+k^{2} h^{2} N_{0}^{2}\right)^{1 / 3} G^{2 / 3}\right]^{3 / 2}\right) .
\end{gathered}
$$

Волновое уравнение, согласно формулам (38), (56) и (58), получается в виде

$$
\begin{gathered}
d^{2} U / d z^{2}+k^{2}\left(N_{0}^{2}+M_{0}^{2} G^{-2 / 3}\right)\left(G+B A^{-1} G^{1 / 3}\right)^{2} \times \\
\times Z_{1 / 3}^{(1) 4}\left(\left(A^{-2 / 3} B+A^{1 / 3} G^{2 / 3}\right)^{3 / 2}\right) U=0
\end{gathered}
$$

а решением его, согласно формулам (40), (58) и (60), является

$$
\begin{gathered}
U=\left(G+B A^{-1} G^{1 / 3}\right)^{-1 / 2}\left[G+\left(B+k^{2} h^{2} M_{0}^{2}\right)\left(A+k^{2} h^{2} N_{0}^{2}\right)^{-1} G^{1 / 3}\right]^{1 / 2} \times \\
\times Z_{1 / 3}^{(1)-1}\left(\left(A^{-2 / 3} B+A^{1 / 3} G^{2 / 3}\right)^{3 / 2}\right) \times \\
\times Z_{1 / 3}\left(\left[\left(A+k^{2} h^{2} N_{0}^{2}\right)^{-2 / 3}\left(B+k^{2} h^{2} M_{0}^{2}\right)+\left(A+k^{2} h^{2} N_{0}^{2}\right)^{1 / 3} G^{2 / 3}\right]^{3 / 2}\right) .
\end{gathered}
$$

В этом примере исключение параметра тоже невозможно, но конечный результат упрощается подстановками

$$
\begin{gathered}
\left(A^{-2 / 3} B+A^{1 / 3} G^{2 / 3}\right)^{3 / 2} \rightarrow G, \\
N_{0}^{2} A^{-1} \rightarrow A^{2}, \\
\left(M_{0}^{2} A-N_{0}^{2} B\right) A^{-5 / 3} \rightarrow B^{2} .
\end{gathered}
$$

После соответствующих выкладок получим вместо (61) и (62) следующие формулы:

$$
d^{2} U / d z^{2}+k^{2}\left(A^{2} G^{2}+B^{2} G^{4 / 3}\right) Z_{1 / 3}^{(1) 4}(G) U=0
$$

и

$$
\begin{gathered}
U=\left(1+k^{2} h^{2} A^{2}+k^{2} h^{2} B^{2} G^{-2 / 3}\right)^{1 / 2} Z_{1 / 3}^{(1)-1}(G) \times \\
\times Z_{1 / 3}\left(\left(1+k^{2} h^{2} A^{2}\right)^{-1}\left[\left(1+k^{2} h^{2} A^{2}\right) G^{2 / 3}+k^{2} h^{2} B^{2}\right]^{3 / 2}\right),
\end{gathered}
$$

тогда как формула (37) примет вид

$$
z / h=Z_{1 / 3}^{(2)}(G) / Z_{1 / 3}^{(1)}(G)
$$

В частном случае $B=0$ получим отсюда формулы $(52)-(54)$ с $\mu=0$, 
$v=1 / 3$. Отметим, что формулы (64)-(66) получаются также, если взять

$$
\begin{aligned}
q(G) & =A+B G, \\
N(G) & =\left(N_{0}^{2}+M_{0}^{2} G\right)^{1 / 2}
\end{aligned}
$$

и сделать в конце надлежащую замену параметра и постоянных.

III. В третьем примере откажемся от условия $f(y)=0$ и положим

$$
\begin{aligned}
& f(y)=-A^{2} y^{4}, \\
& q(G)=1 / 4 G^{2}
\end{aligned}
$$

и

$$
N(G)=\left(N_{0}^{2} G^{\mu-2}-B G^{-2}\right)^{1 / 2}
$$

где $A, B, N_{0}, \mu$ - постоянные. Решения уравнения

$$
d^{2} \Psi / d G^{2}+\left(4 G^{2}\right)^{-1} \Psi=0
$$

выберем в виде

$$
\begin{aligned}
& \Psi_{1}=G^{1 / 2}, \\
& \Psi_{2}=G^{1 / 2} \ln G .
\end{aligned}
$$

Из формул (16) и (19) находим

$$
z / h=K-C^{-1} y^{-1}\left(C-A^{2} y^{2}\right)^{1 / 2}
$$

и

$$
\ln G=A^{-1} \arcsin \left(A y / C^{1 / 2}\right)+\ln H,
$$

где $K$ и $H$ - постоянные. Исключая из этих формул $y$ и выражая $G$, получаем

$$
G=H \exp \left[A^{-1} \arctan \left(A C^{-1}(K-z / h)^{-1}\right)\right] .
$$

В уравнении $(24)-f(y) y^{4}=A^{2}$; следовательно, оно имеет вид

$$
d^{2} \Theta / d G^{2}+\left[k^{2} h^{2} N_{0}^{2} G^{\mu-2}-\left(B k^{2} h^{2}-A^{2}-1 / 4\right) G^{-2}\right] \Theta=0
$$

и его решение есть

$$
\Theta(G)=G^{1 / 2} Z_{2 \mu^{-1}\left(B k^{2} h^{2}-A^{2}\right)^{1 / 2}}\left(2 k h N_{0} \mu^{-1} G^{\mu / 2}\right),
$$

где $Z$ - функция Бесселя. Волновое уравнение (25), исключая с помощью формул (73) и (75) $G$ и $y$, напишем в виде

$$
\begin{gathered}
d^{2} U / d z^{2}+k^{2}\left[C(z / h-K)^{2}+A^{2} / C\right]^{-2} \times \\
\times\left\{N_{0}^{2} H^{\mu} \exp \left[\mu A^{-1} \arctan \left(A C^{-1}(K-z / h)^{-1}\right)\right]-B\right\} U=0 .
\end{gathered}
$$

Согласно формуле (26), его решение таково:

$$
\begin{aligned}
U(z)= & {\left[(z / h-K)^{2}+A^{2} / C\right]^{1 / 2} Z_{2 \mu^{-1}\left(B k^{2} h^{2}-A^{2}\right)^{1 / 2}}\left(2 k h N_{0} \mu^{-1} H^{\mu / 2} \times\right.} \\
& \left.\times \exp \left[(\mu / 2 A) \arctan \left(A C^{-1}(K-z / h)^{-1}\right)\right]\right) .
\end{aligned}
$$

В этом примере, как видим, параметр исключен. Но чтобы сделать конечные формулы более красивыми, введем новые постоянные. Пусть 


$$
\begin{aligned}
& a=\left(C A^{-1}-C^{2}\right)^{1 / 2}, \\
& b=-A-K\left(C A^{-1}-C^{2}\right)^{1 / 2} \\
& c=C \\
& d=(1+b c) a^{-1}=\left(A C^{-1}-A^{2}\right)^{1 / 2}-C K \\
& m=\mu / 4 A \\
& S=B / A^{2} \\
& p=2 N_{0} H^{\mu / 2} \mu^{-1} \exp \left(-(\mu / 2 A) \arccos \left((A C)^{1 / 2}\right)\right) .
\end{aligned}
$$

Тогда волновое уравнение примет вид

$$
\begin{gathered}
d^{2} U / d z^{2}+k^{2}\left[\left(a^{2}+c^{2}\right) z^{2} / h^{2}+2(a b+c d) z / h+\left(b^{2}+d^{2}\right)\right]^{-2} \times \\
\times\left\{4 p^{2} m^{2} \exp \left(4 m \arctan \frac{a z / h+b}{c z / h+d}\right)-S\right\} U=0,
\end{gathered}
$$

a его решение -

$$
\begin{gathered}
U(z)=\left[\left(a^{2}+c^{2}\right) z^{2} / h^{2}+2(a b+c d) z / h+\left(b^{2}+d^{2}\right)\right]^{1 / 2} \times \\
\times Z_{(2 m)^{-1}\left(\left(S h^{2} h^{2}-1\right)^{1 / 2}\right.}\left(k h p \exp \left(2 m \arctan \frac{a z / h+b}{c z / h+d}\right)\right) .
\end{gathered}
$$

Наконец, укажем на связь этого примера с приведенным в $\left[{ }^{3}\right]$ (формулы (33) и (34)). Последний можно рассматривать как вариант данного, получающийся при комплексных значениях некоторых постоянных. Именно, положим

$$
\begin{aligned}
& a \rightarrow(a-c)(1-i) / 2, \\
& b \rightarrow(b-d)(1-i) / 2, \\
& c \rightarrow(a+c)(1+i) / 2, \\
& d \rightarrow(b+d)(1+i) / 2, \\
& m \rightarrow i m,
\end{aligned}
$$

где новые $a, b, c, d, m$ вещественны, причем новые $a, b, c, d$ удовлетворяют, как и старые, соотношению (30). Тогда

$$
\exp \left(4 m \arctan \frac{a z / h+b}{c z / h+d}\right) \rightarrow\left(\frac{a z / h+b}{c z / h+d}\right)^{2 m}
$$

и

$$
\left(a^{2}+c^{2}\right) z^{2} / h^{2}+2(a b+c d) z / h+\left(b^{2}+d^{2}\right) \rightarrow 2 i(a z / h+b)(c z / h+d) .
$$

Если, кроме того, сделаем $S \rightarrow-4 S$, то получим волновое уравнение

$$
d^{2} U / d z^{2}+k^{2}(a z / h+b)^{-2}(c z / h+d)^{-2}\left\{p^{2} m^{2}\left(\frac{a z / h+b}{c z / h+d}\right)^{2 m}-s\right\} U=0,
$$

решение которого таково:

$$
U(z)=(a z / h+b)^{1 / 2}(c z / h+d)^{1 / 2} Z_{(2 m)^{-1}\left(1+4 S h^{2} h^{2}\right)^{1 / 2}}\left(k h p\left(\frac{a z / h+b}{c z / h+d}\right)^{m}\right) .
$$


Эти формулы совпадают с формулами (33) и (34) статьи $\left[{ }^{3}\right]$.

Приведенными тремя примерами мы ограничимся. Вероятно, их можно найти и больше.

\section{ЛИТЕРАТУ РА}

1. Бреховских Л. М., Волны в слоистых средах., Изд. 2-е, М., «Наука», 1973.

2. К а р д П., Изв. АН ЭССР, Физ. Матем., 26, № 3, 252-259 (1977).

3. К а р д П., Изв. АН ЭССР, Физ. Матем., 29, № 1, 1-7 (1980).

4. К а р д П., Изв. АН ЭССР, Физ. Матем., 29, № 2, 113-119 (1980).

5. К а р д П., Изв. АН ЭССР, Физ. Матем., 29, № 3, 336-338 (1980).

6. К а м к е Э., Справочник по обыкновенным дифференциальным уравнениям, Изд. 2-е, М., ГИФМЛ, 1961.

Тартуский государственный университет
Поступила в редакцию 1/XII 1980

\section{P. KARD}

\section{OHEST OHEMOOTMEQLISTE LAINEVORRANDITE LAHENDUSMEETODIST}

Kui $\Psi_{1}(G)$ ja $\Psi_{2}(G)$ on võrrandi (13) lineaarselt sōltumatud lahendid, mille vronskiaan võrdub ühega, ja kui $\Theta(G)$ rahuldab võrrandit (24), siis avaldub ühemōõtmelise lainevõrrandi (25) lahend valemiga (26). Selles vôrrandis on $z$ koordinaat, $k$ lainearv, $G$ ja y parameetrid. Parameetrite vahel kehtib seos (19); koordinaadi sõltuvuse nendest määrab valem (16). Funktsioonide $f(y), q(G)$ ja $N(G)$ valik on meelevaldne, kuid võrrandite (13) ja (24) lahendid peavad olema tuntud. Erijuhul $f(y)=0$ on lainevōrrandil kuju (38) ja tema lahend on (40), kusjuures $\Theta(G)$ rahuldab võrrandit (31) ning seose $z$ ja $G$ vahel annab valem (37). Konkreetsed näited on toodud valemites (52) - (54), (64)-(66) ja (81)-(82), kus $Z$ tähendab Besseli funktsiooni.

\section{P. KARD}

\section{A METHOD FOR SOLVING ONE-DIMENSIONAL WAVE EQUATIONS}

If $\Psi_{1}(G)$ and $\Psi_{2}(G)$ are the linearly independent solutions of the equation (13) with the Wronskian equal to unity, and if $\Theta(G)$ satisfies the equation (24), then the solution of the one-dimensional wave equation (25) is of the form (26). In this equation $z$ is the co-ordinate, $k$ the wave number, $G$ and $y$ parameters. The parameters are connected by the formula (19), and the co-ordinate depends on them via formula (16). The functions $f(y), q(G)$, and $N(G)$ can be chosen arbitrarily, but the equations (13) and (24) must have solutions in terms of the known functions. When $f(y)=0$, the wave equation turns to (38) and its solution to $(40)$, whereas $\Theta(G)$ satisfies the equation (31) and the co-ordinate is connected with the parameter via the formula (37). As examples, three equations with their solutions are established (formulae (52) (54), (64)-(66), and $(81)-(82)$, where $Z$ denotes Bessel functions). 